\title{
SAND99.3220C
}

\section{A Null-Steering Viewpoint of Interferometric SAR}

\author{
D. L. Bickel \\ Sandia National Laboratories \\ Albuquerque, NM 87185-0537 USA \\ Telephone: 505-845-9038, Facsimile: 505-845-9888 \\ Email: dlbicke@sandia.gov
}

\begin{abstract}
Interferometric synthetic aperture radar (IFSAR) extends the two-dimensional imaging capability of traditional synthetic aperture radar to three-dimensions by using an aperture in the elevation plane to estimate the 3-D structure of the target. The operation of this additional aperture can be viewed from a null-steering point of view, rather than the traditional phase determination point of view. Knowing that IFSAR can be viewed from the null-steering perspective allows us to take advantage of the mathematical foundation developed for nullsteering arrays. In addition, in some problems of interest in IFSAR the null-steering perspective provides better intuition and suggests alternative solutions. One example is the problem of estimating building height where layover is present.
\end{abstract}

\section{Introduction}

IFSAR has become an important technology in providing digital elevation models (DEMs). In this paper we examine IFSAR as a beamforming array. In particular, we consider the relationship between IFSAR and null-steering.

Assume an antenna array that is to be used to find the direction of a single target. One way that this target can be located is to electronically scan the antenna beam searching for the direction in which the return is maximum. The beam is steered by applying a linear phase across an antenna array of equally spaced elements. In general, the adjustment of the phases from element to element across the antenna array causes the steering of the beam. We observe that this is related to the IFSAR process. Essentially in IFSAR, we find the phase difference in a two-element antenna array that will provide the maximum return signal. In other words, IFSAR attempts to optimally steer the sum beam of a two-element antenna in the direction of a single target by finding the steering phase to apply to the array to maximize the return. The array in IFSAR typically consists of two phase centers in the elevation plane. As opposed to typical arrays, in IFSAR the spacing of the phase centers are much greater than half of a wavelength.

The antenna array could be used to steer a null at the target rather than pointing the mainlobe at the target. In this case, the direction of the target is chosen such that the return is minimized rather than maximized, as previously described. This null-steering can be thought of as rejecting an unwanted signal such as a jammer, or it can be thought of as a "highresolution" direction-of-arrival method [1].

\section{Null-Steering and IFSAR}

From the IFSAR perspective the single target, or "jammers" if you will, are the clutter cells covered by each rangeDoppler cell. So in IFSAR we wish to steer an independent null for each of the range-Doppler cells. The phase is chosen in such a manner as to attempt to minimize the return energy on the average.

IFSAR can be shown to be a kind of "phase-only" null-steering [2]. The derivation of the phase-only nullsteering presented here follows from [3], [4]. It is also highly related to the derivation in [5]. In null-steering, it is desirable to maximize the ratio of power of the desired output signal to unwanted clutter and noise. This result is achieved by maximizing the normalized improvement factor:

$$
I F=\frac{w^{H} R_{s} w}{w^{H} R_{c} w}
$$

where:

$I F$ - is the normalized improvement factor

$w$ - is the weighting function to accomplish the improvement

$R_{s}$ - is the normalized signal covariance matrix

$R_{c}$ - is the normalized clutter plus noise covariance matrix 
The normalized clutter and noise covariance matrix for a two-element system is:

$$
R_{c}=\left[\begin{array}{ll}
1 & \mu \\
\mu^{*} & 1
\end{array}\right]
$$

where:

$$
\begin{aligned}
& \mu \text { - is the complex coherence between the } \\
& \text { elements due to clutter and noise }
\end{aligned}
$$

It is assumed that there is no a priori information about the direction of the "signal". In this case, the normalized signal covariance matrix becomes the identity matrix. The improvement factor is:

$$
I F=\frac{w^{H} w}{w^{H} R_{c} w}
$$

The phase-only requirement supplies the condition that:

$$
w^{H} w=C
$$

where:

$$
C \text { - is an arbitrary constant chosen to be } 1
$$

It is apparent that the improvement factor is maximized when the denominator of the rhs of equation (2) is minimized subject to the constraint in equation (3). The method of Lagrangian multipliers yields:

$$
P(w)=w^{H} R_{c} w+\sum_{i=1}^{m} \lambda_{i}\left(1-w_{i}^{H} w_{i}\right)
$$

where:

$$
\begin{aligned}
& m-\text { is the number of antenna elements } \\
& \lambda_{i}-\text { is the } i^{\text {th }} \text { Lagrangian multiplier }
\end{aligned}
$$

The minimization of equation (5) results in the well-known eigenvector/eigenvalue equation:

$$
R_{c} w_{i}=\lambda_{i} w_{i}
$$

Equation (5) is minimized by choosing the Lagrangian multiplier as the minimum eigenvalue of $R_{c}$. The result is that the optimal weights, to within a constant amplitude and phase, are:

$$
w=\left(\frac{1}{\sqrt{2}}\right) \cdot\left[\begin{array}{c}
1 \\
-e^{-j \cdot \psi}
\end{array}\right]
$$

where:

$\psi-$ is the argument of the complex coherence $\mu$

It is readily apparent that $\psi$ is the standard IFSAR phase estimated from the maximum-likelihood estimate of the correlation (see [5] for such a derivation). Applying the weights in equation (7) to the two element IFSAR system represents the phase-only null-steering viewpoint of IFSAR processing. Ignoring the phase and amplitude constants, and applying the weights from equation (7) results in the null equation:

$$
2 \cdot j \cdot \sin \left(\frac{2 \cdot \pi}{\lambda} \cdot B \cdot \sin \theta-\frac{\psi}{2}\right)
$$

where:

$$
\begin{aligned}
& B \text { - is the perpendicular component of the } \\
& \text { baseline } \\
& \lambda-\text { is the radar wavelength }
\end{aligned}
$$

The null-steering operation occurs by using the IFSAR phase, $\psi$, to drive the equation ( 8 ) to a zero crossing.

\section{Null-Steering and IFSAR Coherence}

The corresponding optimal improvement factor is given by the reciprocal of the minimum eigenvalue of the normalized clutter and noise matrix:

$$
I F_{o p t}=\frac{1}{1-|\mu|}
$$

Rearranging equation (9) to solve for the magnitude of the coherence yields an interesting result:

$$
|\mu|=1-\frac{1}{I F_{o p t}}
$$

Equation (9) says that the clutter and noise can be cancelled perfectly only if the clutter and noise are perfectly correlated between the two IFSAR channels. Equation (10) says the opposite, that if we can perfectly cancel the clutter and noise, the clutter and noise must have been perfectly correlated between the two channels. Stated another way, the IFSAR coherence can be thought of as the fraction of the power cancelled by the null-steering operation. 


\section{DISCLAIMER}

This report was prepared as an account of work sponsored by an agency of the United States Government. Neither the United States Government nor any agency thereof, nor any of their employees, make any warranty, express or implied, or assumes any legal liability or responsibility for the accuracy, completeness, or usefulness of any information, apparatus, product, or process disclosed, or represents that its use would not infringe privately owned rights. Reference herein to any specific commercial product, process, or service by trade name, trademark, manufacturer, or otherwise does not necessarily constitute or imply its endorsement, recommendation, or favoring by the United States Government or any agency thereof. The views and opinions of authors expressed herein do not necessarily state or reflect those of the United States Government or any agency thereof. 


\section{DISCLAIMER}

Portions of this document may be illegible in electronic image products. Images are produced from the best available original document. 
A couple of simple examples are provided to show the insight that equation (10) brings to IFSAR. The first example is that of correlated clutter in thermal noise. It is obvious that we are only able to cancel the correlated clutter and not the thermal noise. Equation (10) says that the coherence in this case is the very familiar:

$$
|\mu|=\frac{s}{s+n}
$$

where:

$$
\begin{aligned}
& s-\text { is the clutter power } \\
& n-\text { is the thermal noise power }
\end{aligned}
$$

Another case is that of the spatial decorrelation for a uniform target. Figure 1 illustrates this case. A target with a large angular extent cannot be nulled perfectly with a single null. The degree of cancellation depends upon the width of the null and the angular extent of the target. Equation (10) can be used to calculate the also familiar spatial coherence of:

$$
\begin{aligned}
|\mu| & =1-\frac{\int_{\theta_{0} / 2}^{\theta_{0} / 2} 2 \cdot \sigma_{c} \cdot\left|\sin \left(\frac{2 \cdot \pi \cdot B}{\lambda} \theta\right)\right|^{2} d \theta}{\int_{\theta_{0} / 2}^{\beta_{0} / 2} \sigma_{c} d \theta} \\
& =\operatorname{sinc}\left[\frac{2 \cdot \pi \cdot B}{\lambda} \theta_{0}\right]
\end{aligned}
$$

where:

$\sigma_{c}$ - is the clutter power per unit angle

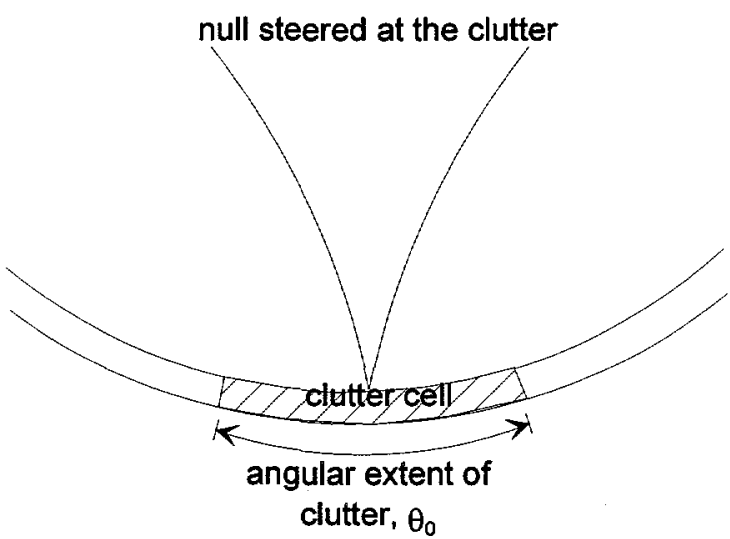

Figure 1: Spatial Decorrelation and Null-Steering

\section{Null-Steering and Ambiguities}

Ambiguities are obvious from the null-steering perspective, just as they are in traditional IFSAR perspective. From the null-steering point of view, ambiguities are the result of repetitive nulls from grating lobes caused by the antenna spacing being larger than half of a wavelength. Figure 2 shows the zero-crossings (nulls) and traditional IFSAR phase wraps.

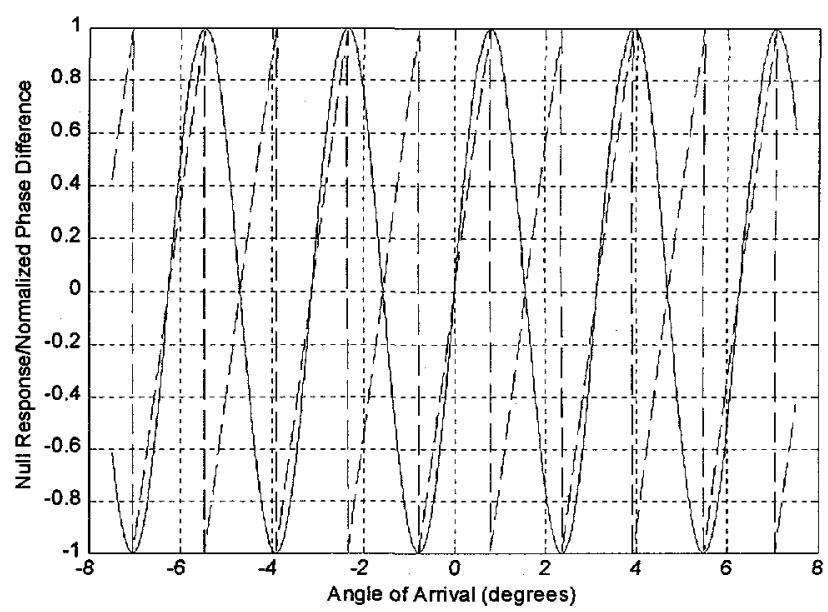

Figure 2: Null Response versus IFSAR Phase Wraps

\section{Null-Steering and the Two Target Case}

In some cases, for example layover of a building, we violate the assumption of a single target in a range-Doppler cell. This case has been evaluated for IFSAR previously using the van Cittert-Zernike theorem [6], [7] However, the nullsteering viewpoint adds intuition into the issue. Figure 3 shows that one of the problems in the two target case is that we have only a single null to steer at two separate targets. It is obvious that we will be unable to cancel both targets, so we will be left with spatial decorrelation in the layover region. The decorrelation gets worse with narrower nulls (larger baselines) and larger target separation (e.g., taller buildings). This result was found in [6], [7] as well. One implication here is that an additional antenna element would allow us to steer a separate null at one of the targets while retrieving the IFSAR phase, or steering another null, at the other target. The additional antenna element would provide enough degrees-of-freedom to find both targets, for example, from the the top and the bottom of the building. Certainly there are challenges in designing systems and processing data to find multiple targets. They are too involved to present in this short format. The important point is that the null-steering viewpoint provides insight into this issue.

Another issue discussed in the previous references was the cosinusoidal nature of the coherence as a function of baseline and target height. Figure 4 shows that this is a result of the ambiguities of the nulls.

In Figure 4a the spatial coherence will be low because the combination of target height and ambiguity spacing is such 
that the targets are not cancelled by the nulls. Figure $4 \mathrm{~b}$ shows the case where the baseline width and the target heights are such that the targets are cancelled with the result that the spatial coherence is high.

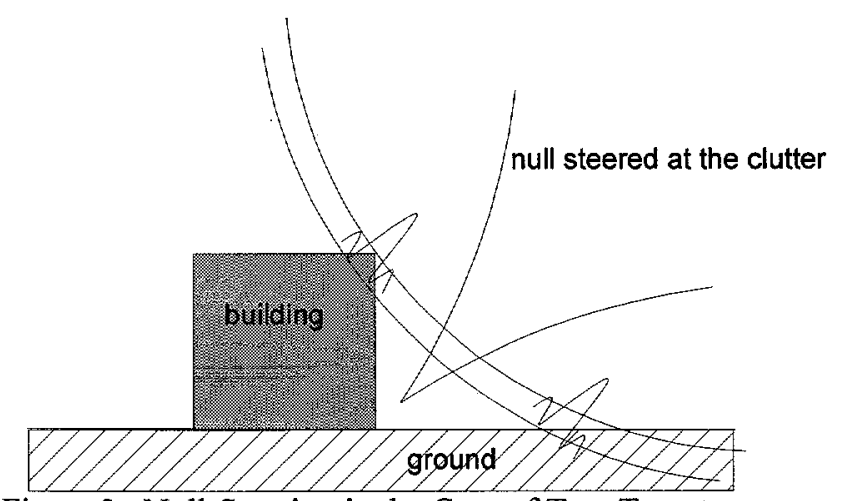

Figure 3: Null-Steering in the Case of Two Targets

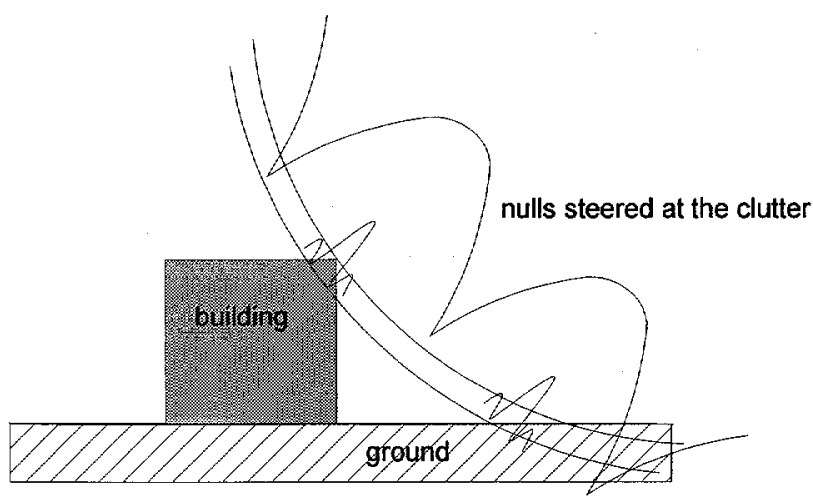

Figure 4a: Low Spatial Coherence Case

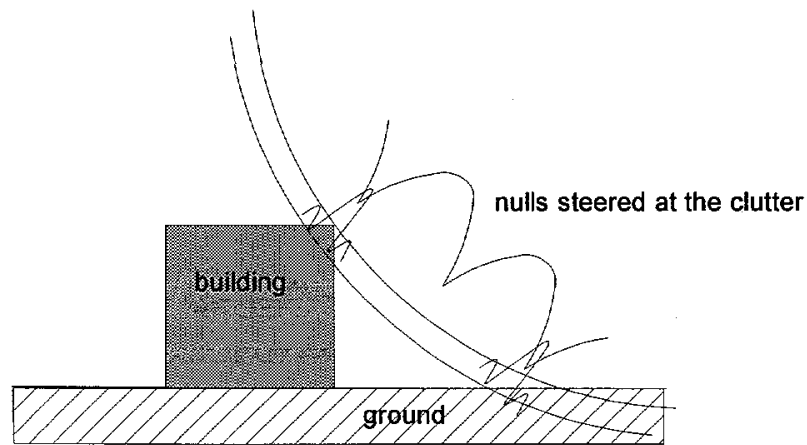

Figure 4b: High Spatial Coherence Case

\section{Conclusions}

In conclusion, we have discussed the strong relationship between phase-only null-steering and IFSAR. The advantage of the null-steering viewpoint is that provides intuition into spatial decorrelation and leads to the concept of using multiple phase centers to begin to separate out multiple targets in layover regions.

\section{Acknowledgments}

This work was supported by the United States Department of Energy under Contract DE-AC04-94AL85000. Sandia is a multiprogram laboratory operated by Sandia Corporation, a Lockheed Martin Company, for the United States Department of Energy.

\section{References}

[1] R. O. Schmidt, R. E. Franks, "Multiple Source DF Signal Processing: An Experimental System", IEEE. Trans. on Antennas and Propagation, Vol. AP-34, No. 3, March, 1986, pp. 281-287.

[2] S. T. Smith, "Optimum Phase-Only Adaptive Nulling", IEEE Trans. on Signal Processing, Vol. 47, No. 7, July, 1999, pp. 1835-1843.

[3] C. A. Baird and G. G. Rassweiler, "Adaptive Sidelobe Nulling Using Digitally Controlled Phase-Shifters", IEEE. Trans on Antennas and Propagation, Vol. AP-24, No. 5, Sept., 1976, pp. 638-649.

[4] D. C. Schleher, MTI and Pulsed Doppler Radar, Artech House, 1991.

[5] C. V. Jakowatz and D. E. Wahl, "Eigenvector method for maximum-likelihood estimation of phase errors in syntheticaperture radar imagery", Journal of the Optical Society of America A, Vol. 10, No. 12, Dec. 1993, pp. 2539-2546.

[6] D. L. Bickel, W. H. Hensley, and D. A. Yocky, "The Effect of Scattering from Buildings on Interferometric SAR Measurements", Proceedings of IGARSS'97, pp. 1545-1547.

[7] D. A. Yocky and C. V. Jakowatz, "Two target height effects on interferometric synthetic aperture radar coherence", Proceedings of SPIE, Vol. 4053, April, 2000. 